\title{
Abnormal learning and forgetting of individual spatial reversal problems in brain-damaged rats
}

\author{
ROBERT THOMPSON \\ Fairview State Hospital, Costa Mesa, California \\ and University of California Irvine Medical Center, Orange, California
}

\begin{abstract}
Except for amygdaloid lesions and cortical cerebellar ablations, selective destruction of virtually any part of the tel-, di-, mes-, or metencephalon was found to impede spatial reversal learning in the rat. (These data contrast sharply with those reported in recent studies on visual reversal learning in the rat.) Test trials given after learning each reversal problem disclosed significant (or marginally significant) deficits in retention only in those groups sustaining damage to the cingulate cortex, ventral hippocampus, rostral caudoputamen, lateral supraoptic hypothalamus, posterolateral hypothalamus, lateral thalamic complex, mediodorsal thalamus, ventromedial thalamus, parafascicular nucleus, substantia nigra, interpeduncular-central tegmental area, pontomesencephalic reticular formation, or pontine reticular formation. It is proposed that there may be at least three classes of learning (amnesic) disorders observed on any given task: (1) a learning impairment only, (2) a retention impairment only, and (3) both a learning and retention impairment.
\end{abstract}

Huppert and Piercy (1979) have presented evidence suggesting that there may be at least two classes of learning (amnesic) disorders in braindamaged patients. In one, learning is impaired, but retention over time of what has been learned is unimpaired. The second is characterized by an impairment (or no impairment) in learning, but retention over time of what has been learned is impaired. Specifically, these investigators compared forgetting curves in H.M. (known to have hippocampal damage) with those in alcoholic Korsakoff patients (presumed to have diencephalic damage) and control subjects. They found that while both H.M. and the Korsakoff patients took longer to learn certain pictorial material than the controls, only H.M. showed rapid forgetting.

These findings led Huppert and Piercy to propose that the first type of learning disorder (learning defect only) is associated with diencephalic lesions, while the second type (retention defect alone or in combination with a learning defect) is associated with hippocampal lesions. Some support for this proposal relative to amnesic patients has recently been reported by Squire (1980).

Interestingly, there is a modest amount of evidence coming from the study of brain-damaged rodents suggesting that the Huppert and Piercy classification of learning disorders may extend to lower mammalian forms. In one study, it was found that, while both hippocampal-damaged rats and

The author is affiliated with the Department of Physical Medicine and Rehabilitation of the University of California Irvine Medical Center. His mailing address is: Fairview State Hospital, 2501 Harbor Boulevard, Costa Mesa, California 92626. mammillary-body-damaged rats took longer to learn individual spatial reversal problems than the controls, only those rats with hippocampal damage showed rapid forgetting (Thompson, 1981). Sara and David-Remacle (1981) have reported that hippocampal-lesioned rats show defective retention of a visual discrimination habit without showing defective learning. A similar pattern of results has been reported by Zornetzer, Gold, and Boast (1977) in connection with learning and retention of a passive avoidance task in hippocampal-lesioned mice.

The foregoing studies seem to establish the existence of at least two classes of learning disorders in brain-damaged rodents. However, the Huppert and Piercy proposal that diencephalic lesions are associated with learning defects alone and that retention defects alone or in combination with learning defects arise only from hippocampal lesions may not apply to lower mammalian forms to the same extent that it applies to amnesic patients. For example, rats with parafascicular thalamic lesions have been observed to show defects in both learning and retention of individual spatial reversal problems (Thompson, Kao, \& Yang, 1981). Similar findings have been reported in rats with lesions to either the rostral caudoputamen or substantia nigra (Thompson \& Yang, 1982).

Despite the lack of correspondence over the locus of the lesions responsible for learning and retention (retentiveness) defects, animal studies bearing upon the Huppert and Piercy notion concerning the dichotomization of learning disorders in brain-damaged subjects should lead to new insights into the neurobiological basis of learning and memory. In the current experiment, data are presented 
on the effects of discrete telencephalic, diencephalic, mesencephalic, and metencephalic lesions on learning and retention of individual spatial reversal problems in albino rats. As expected, certain lesion placements led to learning defects alone, whereas other lesion placements led to learning as well as retention defects.

\section{METHOD}

\section{Subjects}

Adult male albino Sprague-Dawley rats $(n=300)$ were studied. Their weights ranged from 400 to $500 \mathrm{~g}$ at the time of surgery. All animals were maintained on a 12-h light-dark cycle, with lights on at $0600 \mathrm{~h}$, and were trained only during the light phase.

Prior to surgery, all animals were handled periodically and checked for eye disease and middle-ear infection. They were usually housed in gang cages (6-8 rats/cage) containing a constant supply of food pellets and water. Postoperatively, each rat was allowed to recover in a separate medium-size cage, but was subsequently paired with another operated rat at least 1 week prior to original learning.

\section{Surgery}

Under deep chloral hydrate anesthesia $(250 \mathrm{mg} / \mathrm{kg})$, the majority of animals sustained one-stage bilateral cortical or subcortical lesions. (A sham-operated control group of 19 rats was subjected to the same surgical procedures as the experimental groups, except for drilling of the skull and insertion of a lesion electrode or suction tip into the brain.) For cortical and olfactory injuries, the suction method was used. Subcortical injuries were made stereotaxically with reference to a modified version of the Massopust rat atlas (Thompson, 1978). Depending upon the region to be destroyed, a constant anodal current of 1.54.0 $\mathrm{mA}$ for a duration of $10 \mathrm{sec}$ was passed through an implanted stainless steel electrode with $1.0-2.0 \mathrm{~mm}$ of the tip exposed. Details of the surgical procedures have been reported elsewhere (Thompson, 1978).

In most cases, the size and locus of the lesions to different brain sites were intended to be comparable to those reported in earlier studies from my laboratory, which also canvassed many different cortical and subcortical areas with lesions (Thompson, 1978). One exception dealt with the cerebellum: only the cerebellar cortex was aspirated, rather than the entire cerebellum. The only other exceptions involved making somewhat smaller lesions to the globus pallidus, anterior thalamus, and red nucleus.

\section{Apparatus}

An enclosed single-unit T-maze adapted for the use of escapeavoidance of footshock as a motive was employed. The alleys, which measured $15.2 \mathrm{~cm}$ wide and $25.4 \mathrm{~cm}$ high, were covered by a transparent Plexiglas sheet. The startbox was $33.5 \mathrm{~cm}$ long, and the arms were $33.0 \mathrm{~cm}$ long. At the end of each arm was a window through which the rat could enter an endbox $(15.2 \times$ $30.4 \times 25.4 \mathrm{~cm}$ ) on that side. During formal training, a black card mounted on a wooden block was placed against each window. The startbox and arms of the T had a grid floor; each endbox had a wooden floor. The grid floor of the apparatus was connected to a Variac, which delivered footshocks with an average intensity of $1.5 \mathrm{~mA}$.

The apparatus was housed in a sound-attenuated room that was illuminated by conventional ceiling fluorescent lights. No effort was made to control for intramaze olfactory cues or any extramaze cues.

\section{Procedure}

After a 2-3-week recovery period, the brain-damaged and sham-operated control rats were habituated to the left and right endboxes and subsequently were trained to run from the startbox, through one of the arms of the $T$, to push aside a black card blocking the window, and to enter the endbox in order to escape from (or avoid) footshock. During this period, initial choices of each arm were monitored in order to determine the side of the $T$ preferred by each animal.

On the following day, all animals were started on a series of eight reversal problems, one reversal problem every $48 \mathrm{~h}$. The first reversal (sometimes termed "original learning") consisted of training the animals to their nonpreferred side, the second reversal consisted of training the animals to the side that was incorrect $48 \mathrm{~h}$ previously, and so on. The correct arm led to an endbox which could be entered by displacing the unlocked card. The incorrect arm, on the other hand, led to a locked card which prevented the animal from entering the endbox on that side. If the incorrect arm was chosen, the animal had to return to the choice-point and choose the other arm of the T. An error, which consisted of an approach response to within $10.8 \mathrm{~cm}$ of the locked card, was automatically punished by footshock. The animal was allowed to remain in the endbox for $10 \mathrm{sec}$, after which it was transferred to a restraining cage to await the next trial. With an intertrial interval of $50-75 \mathrm{sec}$, training to the correct arm was continued in one session until the animal reached the criterion of learning. The criterion of learning was defined as the first appearance of a "perfect" (Grant, 1946) or "nearperfect" (Runnels, Thompson, \& Runnels, 1968) run of correct responses having a probability of occurrence of less than .05. (This type of criterion is particularly suitable when investigating retentiveness of a habit in two or more groups of subjects that differ significantly from each other in the rate of original learning - see Thompson et al., 1981).

Immediately prior to training on Reversal 2 through 8 (and $48 \mathrm{~h}$ after learning the eighth reversal), a single test trial of retention was given. A correct response consisted of choosing the arm that had been correct $48 \mathrm{~h}$ earlier. Errors on test trials were punished by footshock.

\section{Histology}

At the conclusion of the experiment, each brain-damaged rat was killed with an overdose of chloral hydrate. Its vascular system was then perfused with normal saline followed by $10 \%$ Formalin; the brain was removed and stored in 10\% Formalin for 2-4 days. Cortical injuries were reconstructed on Lashley-type brain diagrams. With a freezing microtome, each brain was sectioned frontally at $90 \mu$. Every fourth section showing the lesion was subsequently photographed at $10 \times$ by using the section as a negative film in an enlarger (see Thompson, 1978).

\section{Statistical Analysis}

In this study, the overall reversal-learning score for each animal was expressed as mean errors (including any that may have been committed within the criterion run) per reversal. The overall reversal retention score for each animal was expressed as percentage correct responses over the eight retention-test trials.

The differences in the mean learning and retention scores between each brain-damaged group and the control group were evaluated by the Mann-Whitney $U$ test (two-tailed), corrected for ties.

\section{RESULTS}

\section{Learning}

Overall reversal performance. As expected from an earlier study (Thompson \& Langer, 1963), avoidance of the arm of a single-unit T-maze from which footshock was received $50-75 \mathrm{sec}$ previously is an easy task for the albino rat. It will be noted in Table 1 that all control animals averaged fewer than 2 errors/reversal, a performance level that approximates one-trial learning. 
Table 1

Mean Learning Errors/Reversal, Mean Percentage Correct Responses on Retention Test Trials, and Rank-Order Correlations Between Individual Learning Errors and Retention Errors for All Groups

\begin{tabular}{|c|c|c|c|c|c|c|}
\hline \multirow[b]{2}{*}{ Group } & \multirow[b]{2}{*}{$\mathrm{N}$} & \multicolumn{2}{|c|}{ Learning } & \multicolumn{2}{|c|}{ Retention } & \multirow{2}{*}{$\begin{array}{c}\text { Correla } \\
\text { tion }\end{array}$} \\
\hline & & Mean & Range & Mean & Range & \\
\hline Control & 19 & 1.3 & $1.0-1.8$ & 90.8 & $75-100$ & +.39 \\
\hline Peripheral Blinding & 8 & $1.5^{*}$ & $1.3-1.9$ & 98.4 & $87.5-100$ & +.13 \\
\hline Olfactory Bulb & 7 & $1.9 *$ & $1.1-3.6$ & 89.3 & $75-100$ & +.30 \\
\hline Blind and Olfactory Bulb & 6 & $5.4^{*}$ & $3.4-7.4$ & $62.5^{*}$ & $50-75$ & +.11 \\
\hline \multicolumn{7}{|l|}{ Neocortex } \\
\hline Occipital & 9 & $2.5^{*}$ & $1.6-3.4$ & 87.5 & $75-100$ & +.17 \\
\hline Parietal & 8 & $2.3^{*}$ & $1.8-3.6$ & 89.0 & $75-100$ & +.38 \\
\hline Frontal & 8 & $3.4^{*}$ & $1.3-8.4$ & 84.4 & $62.5-100$ & -.18 \\
\hline \multicolumn{7}{|l|}{ Other Telencephalic Areas } \\
\hline Anterior Cingulate & 10 & $3.9^{*}$ & $1.9-9.9$ & 80.0 & $37.5-100$ & +.32 \\
\hline Posterior Cingulate & 9 & $6.4^{*}$ & $4.9-9.3$ & 79.2 & $50-100$ & +.48 \\
\hline Dorsal Hippocampus & 10 & $4.9^{*}$ & $2.5-7.3$ & 83.8 & $50-100$ & +.14 \\
\hline Ventral Hippocampus & 8 & $1.7 *$ & $1.1-2.5$ & 82.8 & $62.5-100$ & -.45 \\
\hline Septofornix & 7 & $8.9^{*}$ & $6.1-13.5$ & 92.9 & $75-100$ & +.29 \\
\hline Nucleus Accumbens & 7 & $1.9 *$ & 1.14 .4 & 92.9 & $75-100$ & +.03 \\
\hline Amygdala & 6 & 1.3 & $1.1-2.0$ & 95.9 & $87.5-100$ & +.09 \\
\hline Globus Pallidus & 8 & $1.7 *$ & $1.3-1.9$ & 84.4 & $62.5-100$ & +.43 \\
\hline \multicolumn{7}{|l|}{ Thalamus } \\
\hline Anterior & 7 & $6.7^{*}$ & $3.9-11.6$ & 83.9 & $75-100$ & +.68 \\
\hline Mediodorsal & 12 & $6.3^{*}$ & $1.4-22.9$ & $81.2^{*}$ & $50-100$ & -.10 \\
\hline Ventromedial & 9 & $4.3^{*}$ & $1.5-8.5$ & 81.9 & $50-100$ & +.08 \\
\hline Ventrolateral & 7 & $1.6^{*}$ & $1.3-2.3$ & 96.4 & $87.5-100$ & +.12 \\
\hline Lateral & 8 & $2.6^{*}$ & $1.1-5.4$ & $79.7 *$ & $50-100$ & +.10 \\
\hline Ventrobasal & 8 & $1.8^{*}$ & $1.1-3.9$ & 85.9 & $50-100$ & +.40 \\
\hline Nucleus Posterior & 7 & $1.7^{*}$ & $1.1-2.6$ & 92.5 & $75-100$ & +.20 \\
\hline Subthalamus & 7 & $1.5^{*}$ & $1.1-2.0$ & 84.6 & $62.5-100$ & +.25 \\
\hline \multicolumn{7}{|l|}{ Hypothalamus } \\
\hline Medial Supraoptic & 6 & $2.8^{*}$ & $1.6-6.4$ & 89.6 & $62.5-100$ & +.69 \\
\hline Lateral Supraoptic & 7 & $2.7 *$ & $1.8-5.3$ & 78.6 & $62.5-100$ & +.43 \\
\hline Ventromedial & 7 & $3.5^{*}$ & $2.0-6.1$ & 89.3 & $75-100$ & -.03 \\
\hline Posterolateral & 7 & $4.8 *$ & $1.5-7.6$ & $71.4^{*}$ & $37.5-87.5$ & +.57 \\
\hline Mammillary Bodies & 7 & $6.0^{*}$ & $5.0-7.3$ & 87.5 & $62.5-100$ & -.52 \\
\hline \multicolumn{7}{|l|}{ Brainstem Areas } \\
\hline Pretectum & 7 & $1.5^{*}$ & $1.1-2.0$ & 92.9 & $75-100$ & +.21 \\
\hline Superior Colliculus & 6 & $2.1 *$ & $1.6-2.8$ & 87.5 & $75-100$ & +.10 \\
\hline Central Gray & 8 & $2.0^{*}$ & $1.4-2.8$ & 87.5 & $62.5-100$ & +.62 \\
\hline Red Nucleus & 10 & $1.6^{*}$ & $1.1-2.0$ & 93.7 & $75-100$ & -.31 \\
\hline Central Tegmentum & 7 & $2.9^{*}$ & $2.0-3.9$ & $71.4^{*}$ & $25-100$ & -.28 \\
\hline Median Raphe & 6 & $6.5^{*}$ & $3.1-13.9$ & 89.6 & $75-100$ & -.77 \\
\hline Dorsolateral Midbrain RF & 6 & $1.6^{*}$ & $1.1-2.0$ & 91.7 & $75-100$ & -.21 \\
\hline Pontomesencephalic RF & 7 & $4.6^{*}$ & $1.9-9.8$ & $75.0^{*}$ & $62.5-100$ & +.68 \\
\hline Pontine RF & 6 & $5.2 *$ & $1.9-9.0$ & 79.2 & $62.5-100$ & +.75 \\
\hline Cerebellar Cortex & 8 & 1.3 & $1.0-1.5$ & 93.8 & $75-100$ & +.54 \\
\hline
\end{tabular}

Note-RF = reticular formation. * Differed from the controls at least at the .05 level.

As shown in Table 1, 35 of the 37 brain-damaged groups examined in this study were significantly inferior to the controls in acquiring the series of eight reversal problems. Those groups demonstrating the greatest disturbance in overall reversal learning (averaging at least 3.5 errors/reversal) had suffered a combination of enucleation of the eyes and olfactory bulbectomy or had received discrete lesions to the anterior cingulate cortex, posterior cingulate cortex, dorsal hippocampus, septofornix, anterior thalamus, mediodorsal thalamus, ventromedial thalamus, ventromedial hypothalamus, posterolateral hypothalamus, mammillary bodies, median raphe, pontomesencephalic reticular for- mation, or pontine reticular formation. The only groups that were not significantly inferior to the controls in overall reversal learning had sustained damage to the amygdaloid complex or cerebellar cortex. Figures 1-5 show representative lesions sustained by the animals of the foregoing groups.

It is interesting to note that those rats rendered blind by enucleation or anosmic by olfactory bulbectomy showed only a slight deficit in overall reversal learning-the difference between the two groups in error scores fell considerably short of statistical significance. However, those rats rendered both blind and anosmic were profoundly impaired in learning the series of eight reversals, and com- 


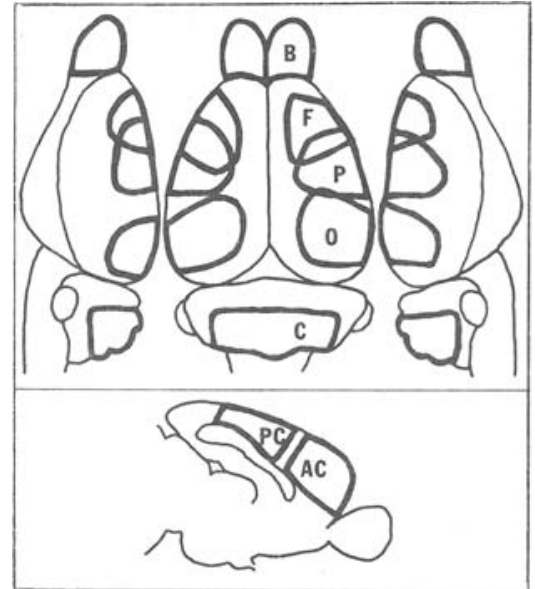

Figure 1. Schematic drawings of the dorsal and lateral surface of the rat brain (upper panel) and a parasagittal section of the rat brain (lower panel), showing the region of common damage (areas enclosed by heavy solid lines) sustained by the olfactory bulb (B), frontal (F), parietal (P), occipital (O), cerebellar (C), anterior cingulate (AC), and posterior cingulate (PC) groups.

mitted significantly more errors/reversal than either the blinded group or the anosmic group (ps $<.01$ ).

Of equal interest is the finding that there appears to be no critical neocortical focus for spa-

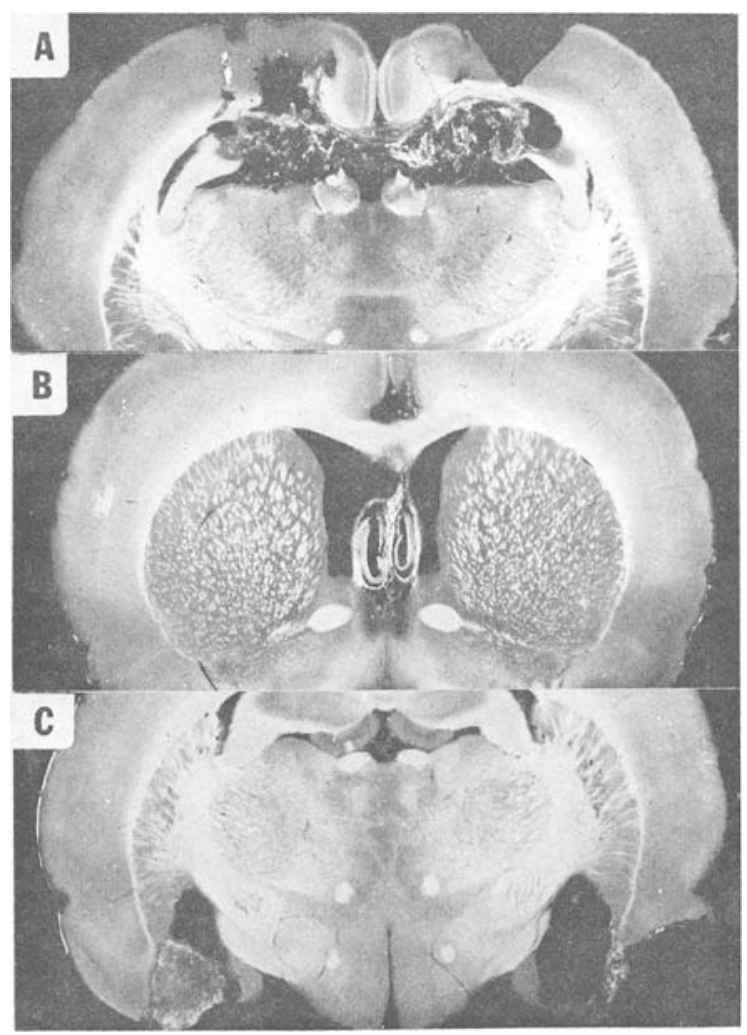

Figure 2. Photographs of unstained sections (from three animals) showing lesions to the dorsal hippocampus (A), septofornix (B), and amygdala (C). tial reversal-learning deficits in the albino rat (see Figure 1, top panel). This is suggested by the fact that, although the occipital (mean cortical damage $=29.2 \%$, range $=26 \%$ to $33 \%$ ), parietal (mean cortical damage $=25.5 \%$, range $=17 \%$ to $30 \%$ ), and frontal (mean cortical damage $=21.8 \%$, range $=$ $15 \%$ to $31 \%$ ) groups were impaired in overall reversal learning, none of the intergroup differences approached statistical significance. Furthermore, a mass-action effect was not obtained; a nonsignificant rank-order correlation of +.13 was computed between the extent of neocortical damage and the number of reversal-learning errors for all 25 neocortically damaged rats. Nonsignificant correlations were likewise obtained for the respective occipital $(\mathrm{rho}=+.36)$, parietal $($ rho $=-.11)$, and frontal (rho $=+.35$ ) groups.

Other intergroup comparisons showed that greater reversal-learning deficits were produced by occipital lesions than by enucleation of the eyes $(p<$ .05 ), by posterior cingulate lesions than by anterior cingulate lesions $(p<.05)$, by dorsal hippocampal lesions than by ventral hippocampal lesions $(p<.01)$, by dorsomedial or ventromedial thalamic lesions than by ventrolateral or lateral thalamic lesions ( $\mathrm{ps}<.05$ ), by posterolateral hypothalamic lesions than by lateral supraoptic le-

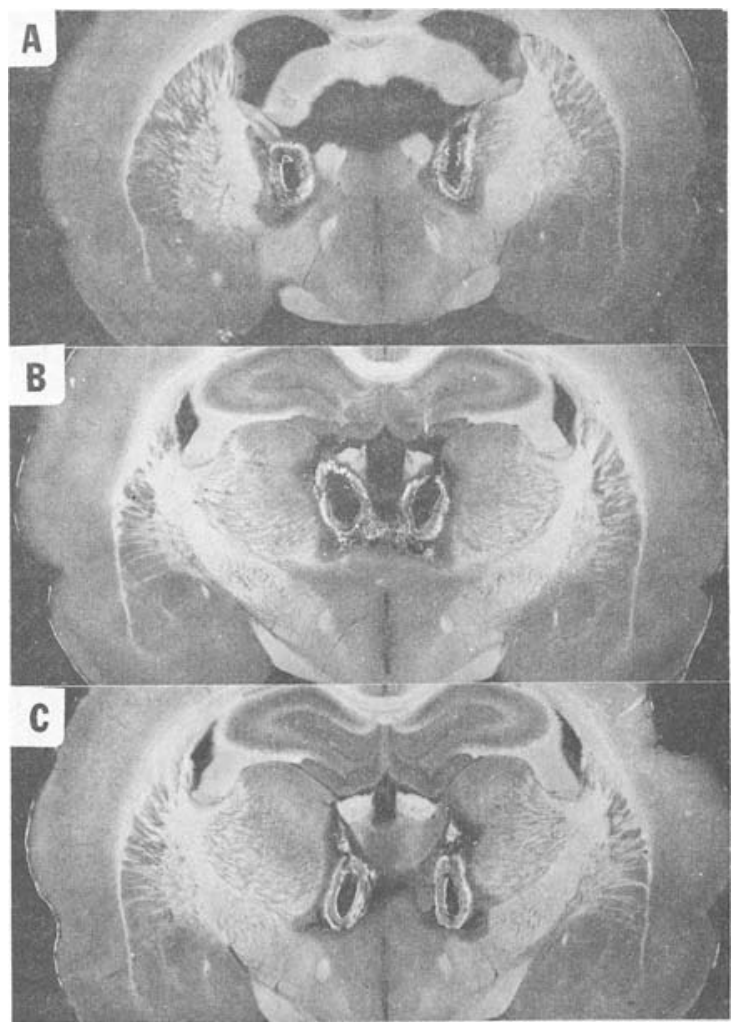

Figure 3. Photographs of unstained sections (from three animals) showing lesions to the anterior thalamus (A), mediodorsal thalamus (B), and ventromedial thalamus (C). 


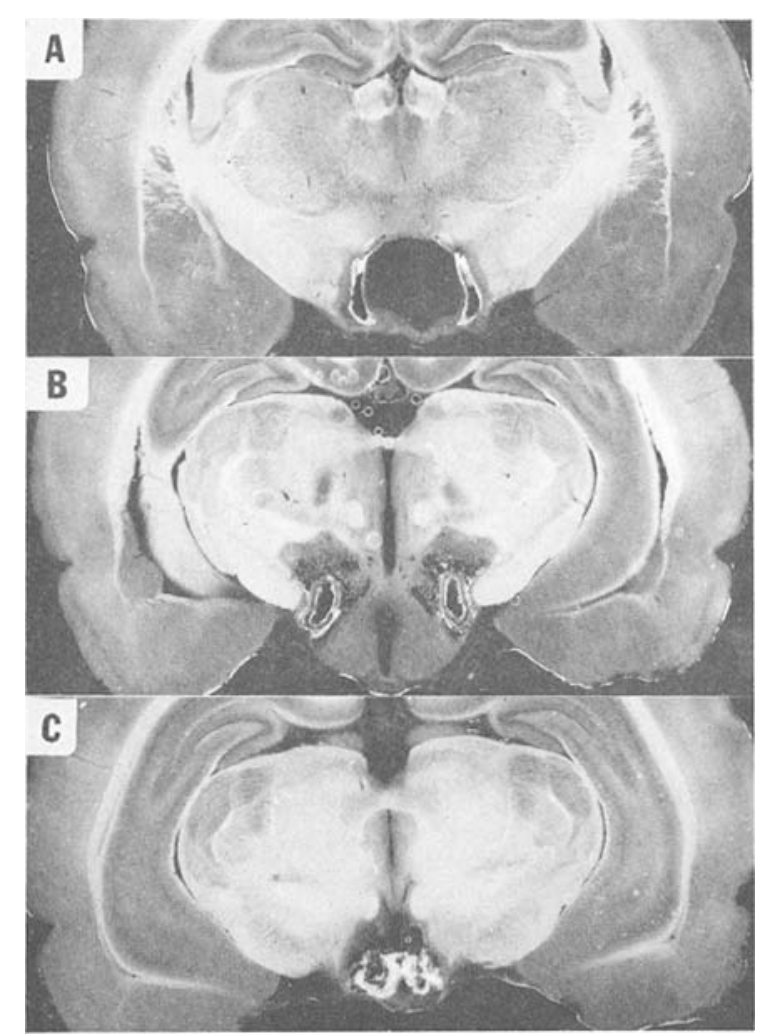

Figure 4. Photographs of unstained sections (from three animals) showing lesions to the ventromedial hypothalamus (A), posterolateral hypothalamus (B), and mammillary bodies $(C)$.

sions $(\mathrm{p}<.05)$, by mammillary body lesions than by ventromedial hypothalamic or medial supraoptic lesions (ps $<.05$ ), and by ventral pontine or ventral pontomesencephalic reticular formation lesions than by dorsomedial mesencephalic reticular formation lesions (ps $<.01)$.

First reversal versus second reversal. As seen in Table $2,{ }^{1}$ the controls performed as efficiency on the second reversal problem as they did on the first reversal problem. With respect to the brain-damaged groups, there is a suggestion that the first reversal (or original learning) problem is more sensitive to the presence of brain damage than is the subsequent reversal problem. Of the 37 experimental groups shown in Table 2, 34 exhibited a significant deficit on the first reversal. While none of the three groups (those failing to show a deficit on the first reversal) exhibited a deficit on the second reversal, 6 of the $\mathbf{3 4}$ groups (those showing a deficit on the first reversal) failed to exhibit a deficit on the second reversal. Based on the binomial test (see Siegel, 1956), this was a significant change $(p<.05)$.

First four reversals versus last four reversals. All brain-damaged groups and the control group tended to make fewer errors on the last four reversals than they did on the first four reversals (see Table 2). In 19 instances, this improvement in reversal performance was significant.

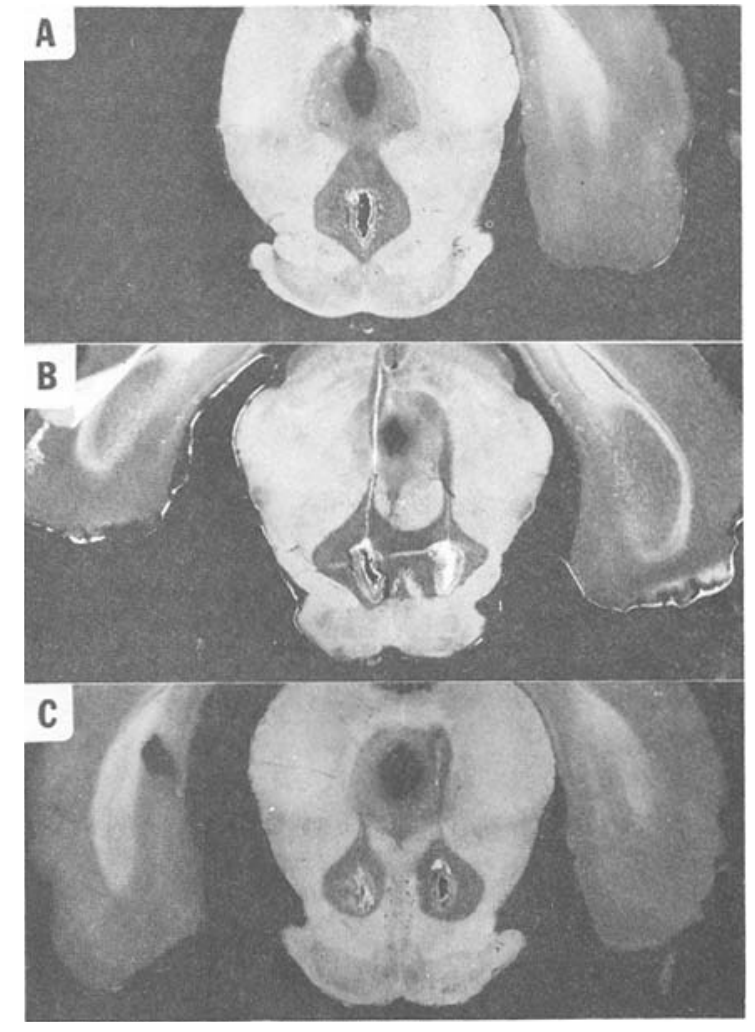

Figure 5. Photographs of unstained sections (from three animals) showing lesions to the median raphe (A), pontomesencephalic reticular formation (B), and pontine reticular formation (C).

\section{Retention.}

On the whole, the controls showed little forgetting of the individual spatial reversal problems over a 48-h period (see Table 1). Ten of the sham-operated animals failed to make a single error during the course of the experiment, four made one error, and five made two errors.

Of the 37 brain-damaged groups, only 6 made significantly more errors on the retention test than the controls. They suffered either a combination of peripheral blinding and olfactory bulbectomy or discrete lesions to the mediodorsal thalamus (Figure 3B), lateral thalamus (Figure 6A), posterolateral hypothalamus (Figure 4B), interpeduncularcentral tegmental complex (Figure 6B), or pontomesencephalic reticular formation (Figure 5B). Six additional groups showed a marginally significant $(p=.10)$ deficit in retention. Their lesions involved the anterior cingulate cortex (Figure 1, bottom panel), posterior cingulate cortex (Figure 1, bottom panel), ventral hippocampus (Figure 7A), ventromedial thalamus (Figure $3 C$ ), lateral supraoptic hypothalamus (Figure 7B), or pontine reticular formation (Figure 5C).

In contrast to reversal learning, performance on the retention test did not improve over the course of training. In fact, the control group as well as 28 of the 37 brain-damaged groups showed a de- 
Table 2

Mean Learning Errors on First Reversal vs. Second Reversal and Mean Learning Errors/Reversal on First Four Reversals vs. Last Four Reversals for All Groups

\begin{tabular}{|c|c|c|c|c|c|c|}
\hline \multirow[b]{2}{*}{ Group } & \multicolumn{6}{|c|}{ Reversal } \\
\hline & First & Second & Difference & First Four & Last Four & Difference \\
\hline Control & 1.3 & 1.3 & & 1.5 & 1.1 & $.4 \dagger$ \\
\hline Peripheral Blinding & 1.9 & 1.3 & $.6 \dagger$ & 1.5 & 1.4 & .1 \\
\hline Olfactory Bulb & $3.0^{*}$ & $2.7 *$ & .3 & 2.2 & 1.6 & $.6 \dagger$ \\
\hline Blind and Olfactory Bulb & $6.0^{*}$ & $9.0 *$ & $-3.0 \dagger$ & $7.4^{*}$ & $3.3^{*}$ & $4.1 \dagger$ \\
\hline \multicolumn{7}{|l|}{ Neocortex } \\
\hline Occipital & $3.3^{*}$ & $4.8^{*}$ & -1.5 & $3.3^{*}$ & $1.7 *$ & $1.6 \dagger$ \\
\hline Parietal & $3.0^{*}$ & $3.4^{*}$ & -.4 & $2.8^{*}$ & $1.8^{*}$ & $1.0 \dagger$ \\
\hline Frontal & $3.1^{*}$ & $5.5^{*}$ & -2.4 & $3.6^{*}$ & $3.2 *$ & .4 \\
\hline \multicolumn{7}{|l|}{ Other Telencephalic Areas } \\
\hline Anterior Cingulate & $3.2^{*}$ & $5.6^{*}$ & -2.4 & $4.1^{*}$ & $3.7 *$ & .4 \\
\hline Posterior Cingulate & $9.0^{*}$ & $7.3^{*}$ & 1.7 & $7.9^{*}$ & $4.9 *$ & 3.0 \\
\hline Dorsal Hippocampus & $8.3^{*}$ & $8.1^{*}$ & .2 & $6.7^{*}$ & $3.1 *$ & $3.6 \dagger$ \\
\hline Ventral Hippocampus & $2.6^{*}$ & $2.0^{*}$ & .6 & $2.0^{*}$ & 1.4 & .6 \\
\hline Septofornix & $10.0 *$ & $10.0 *$ & & $10.4 *$ & $7.4^{*}$ & $3.0 \dagger$ \\
\hline Nucleus Accumbens & $2.0 *$ & $2.4 *$ & -.4 & 2.1 & 1.9 & .2 \\
\hline Amygdala & 1.8 & 2.2 & -.4 & 1.6 & 1.0 & .6 \\
\hline Globus Pallidus & $2.0^{*}$ & $2.6^{*}$ & $-.6 \dagger$ & $2.0^{*}$ & 1.4 & $.6 \dagger$ \\
\hline \multicolumn{7}{|l|}{ Thalamus } \\
\hline Anterior & $5.9 *$ & $7.7 *$ & -1.8 & $9.2 *$ & $4.2^{*}$ & $5.0 \dagger$ \\
\hline Mediodorsal & $7.3^{*}$ & $7.5^{*}$ & -.2 & $7.0 *$ & $5.6^{*}$ & 1.4 \\
\hline Ventromedial & $5.4^{*}$ & $4.0^{*}$ & 1.4 & $4.6^{*}$ & $4.0 *$ & .6 \\
\hline Ventrolateral & $2.1 *$ & $2.0 *$ & .1 & $2.0 *$ & 1.2 & $.8 \dagger$ \\
\hline Lateral & $6.6^{*}$ & $2.3 *$ & $4.3 \dagger$ & $3.3^{*}$ & $1.9 *$ & $1.4 \dagger$ \\
\hline Ventrobasal & $3.3^{*}$ & 2.3 & 1.0 & 2.3 & 1.3 & 1.0 \\
\hline Nucleus Posterior & $2.7^{*}$ & 2.0 & .7 & 2.0 & 1.3 & .7 \\
\hline Subthalamus & $2.3^{*}$ & 1.6 & .7 & 1.6 & 1.4 & .2 \\
\hline \multicolumn{7}{|l|}{ Hypothalamus } \\
\hline Medial Supraoptic & $3.8^{*}$ & $5.0 *$ & -1.2 & $3.5^{*}$ & $2.1^{*}$ & 1.4 \\
\hline Lateral Supraoptic & $5.1 *$ & $3.4^{*}$ & 1.7 & $3.6^{*}$ & 1.8 & $1.8 \dagger$ \\
\hline Ventromedial & $7.3 *$ & $5.3 *$ & 2.0 & $4.5^{*}$ & $2.5^{*}$ & $2.0 \dagger$ \\
\hline Posterolateral & $6.3 *$ & $5.3 *$ & 1.0 & $6.0 *$ & $3.6^{*}$ & $2.4 \dagger$ \\
\hline Mammillary Bodies & $8.6^{*}$ & $6.4^{*}$ & 2.2 & $7.0 *$ & $5.0^{*}$ & 2.0 \\
\hline \multicolumn{7}{|l|}{ Brainstem Areas } \\
\hline Pretectum & $2.6^{*}$ & 1.3 & $1.3 \dagger$ & 1.7 & 1.3 & .4 \\
\hline Superior Colliculus & $3.3^{*}$ & 1.7 & 1.6 & $2.5^{*}$ & $1.7^{*}$ & .8 \\
\hline Central Gray & $3.0^{*}$ & $3.9 *$ & -.9 & $2.6^{*}$ & 1.4 & $1.2 \dagger$ \\
\hline Red Nucleus & $2.8^{*}$ & $2.0^{*}$ & $.8 \dagger$ & $2.0^{*}$ & 1.2 & $.8 \dagger$ \\
\hline Central Tegmentum & $4.9^{*}$ & $2.9 *$ & 2.0 & $3.7 *$ & $2.1^{*}$ & $1.6 \dagger$ \\
\hline Median Raphe & $8.8 *$ & $4.8^{*}$ & 4.0 & $8.8 *$ & $4.2 *$ & $4.6 \dagger$ \\
\hline Dorsolateral Midbrain RF & $3.3 *$ & 1.3 & $2.0 \dagger$ & 1.9 & 1.3 & $.6 \dagger$ \\
\hline Pontomesencephalic RF & $5.7^{*}$ & $7.6 *$ & -1.9 & $4.9 *$ & $4.3^{*}$ & .6 \\
\hline Pontine RF & $6.0^{*}$ & $5.2 *$ & .8 & $6.0 *$ & $4.4^{*}$ & 1.6 \\
\hline Cerebellar Cortex & 1.9 & 1.4 & .5 & 1.4 & 1.2 & .2 \\
\hline
\end{tabular}

Note-RF = reticular formation.

(Wilcoxon test).

*Differed from the controls at least at the .05 level.

tSignificant at least at the .05 level

cline in retention scores on the last four test trials as compared with the first four test trials. In no case, however, did these differences reach statistical significance.

One interesting observation concerns the group subjected to enucleation of the eyes. This group made no errors on the last four retention test trials, a performance level that significantly surpassed that of the control group $(p=.05)$.

\section{Learning Scores Versus Retention Scores}

The control group disclosed a slight, but marginally significant $(p=.10)$, positive relationship between the number of learning errors/reversal and the number of retention errors (see Table 1). None of the rank-order correlations for the remaining 37 groups approached statistical significance, although the majority were in the positive direction.

\section{Summary}

Except for amygdaloid lesions and cortical cerebellar ablations, selective destruction of virtually any part of the tel-, di-, mes-, or metencephalon was found to impede spatial reversal learning in the rat. Therefore, mapping the individual critical brain sites for spatial reversal learning was arbitrarily based on the lesion placements in those experimental groups averaging at least 2.6 errors/reversal. 


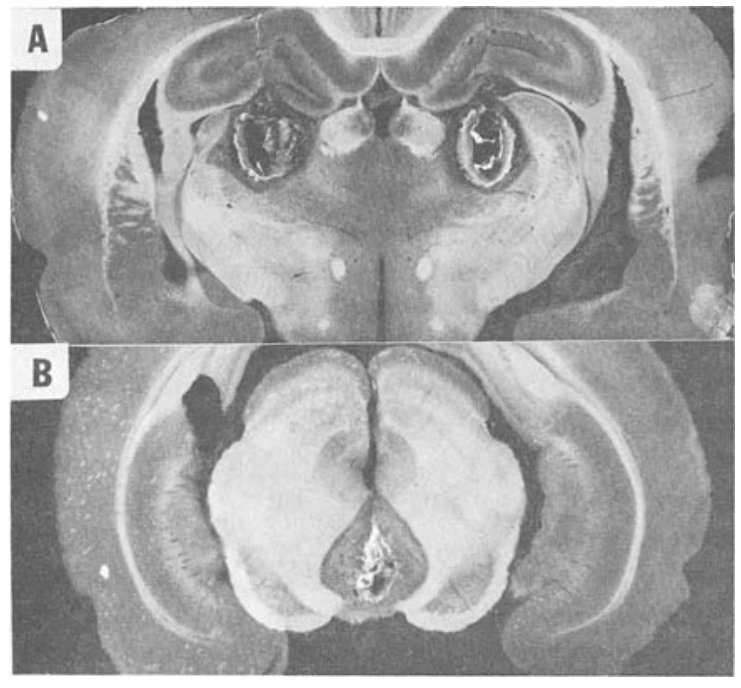

Figure 6. Photographs of unstained sections (from two animals) showing lesions to the lateral thalamic complex (A) and interpeduncular-central tegmental area (B).

These sites are designated by capital letters in Figure 8. To emphasize possible foci among these critical areas, encircled capital letters indicate lesion placements in those groups averaging at least $4.3 \mathrm{er}$ rors/reversal.

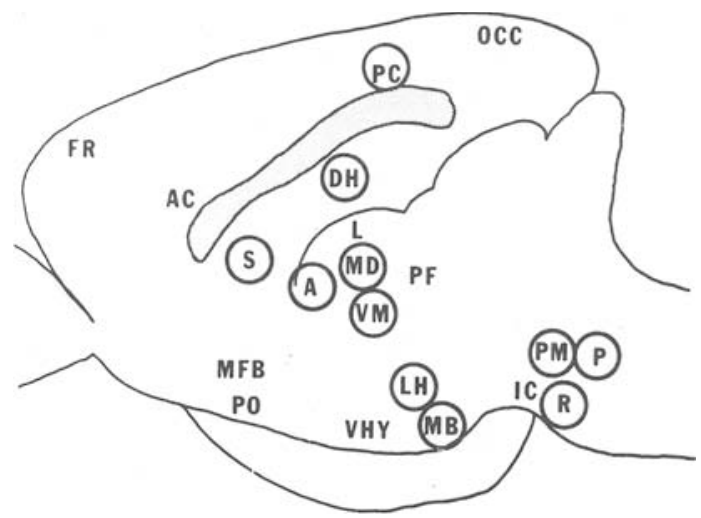

Figure 8. Schematic drawing of a parasagittal section of the rat brain showing the relative positions of those lesion placements producing moderate (unencircled capital letters) and severe (encircled capital letters) reversal-learning deficits. Abbreviations: $\mathbf{A}=$ anterior thalamus; $\mathbf{A C}=$ anterior cingulate cortex; CG = mesencephalic central gray; $D H=$ dorsal hippocampus; $\mathbf{F R}=$ frontal (motor) cortex; $\mathbf{G P}=$ globus pallidus; $\mathbf{I C}=$ interpeduncular-central tegmental area; $\mathbf{L}=$ lateral thalamic complex; $\mathbf{L H}=$ posterolateral hypothalamus; $\mathbf{M B}=$ mammillary bodies; MD = mediodorsal thalamus; $M F B=$ lateral supraoptic hypothalamus; $O C C=$ occipital cortex; $P=$ pontine reticular formation; $\mathbf{P C}=$ posterior cingulate cortex; $\mathbf{P F}=$ parafascicular nucleus; $\mathbf{P M}=$ pontomesencephalic reticular formation; $\mathbf{P O}=$ medial supraoptic hypothalamus; $R=$ median raphe; $R C P=$ rostral caudoputamen; $R N=$ red nucleus; $S=$ septofornix; SN = substantia nigra; VH = ventral hippocampus; VHY = ventromedial hypothalamus; $V L=$ ventrolateral thalamus; VM = ventromedial thalamus.

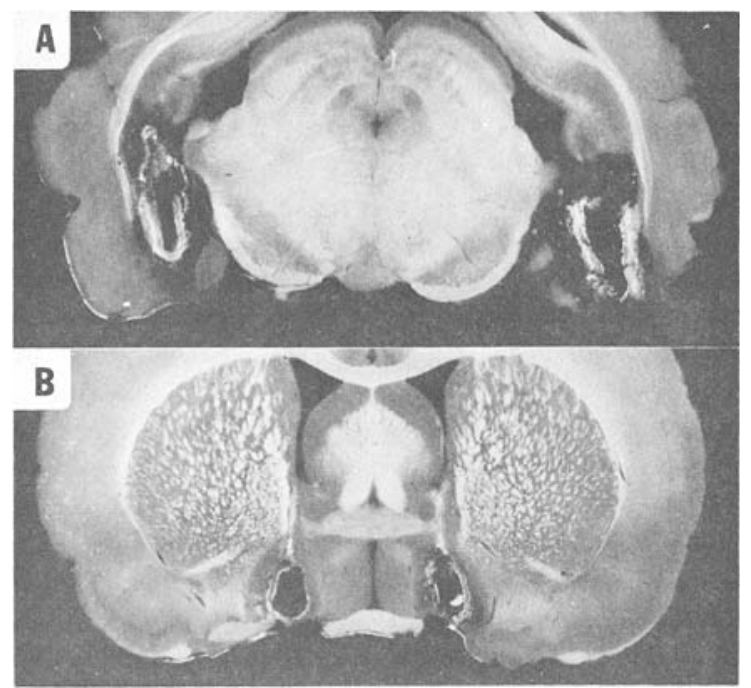

Figure 7. Photographs of unstained sections (from two animals) showing lesions to the ventral hippocampus (A) and lateral supraoptic hypothalamus (B).

The critical brain sites for spatial reversal retention are depicted in Figure 9. Unencircled capital letters refer to lesion placements in those groups showing a marginally significant deficit, while encircled capital letters refer to lesion placements in those groups showing a significant deficit.

For comparative purposes, the data on the rostral caudoputamen, parafascicular nucleus, and substantia nigra (Thompson et al., 1981; Thompson \& Yang, 1982) are included within Figures 8 and 9; the experimental procedures used to study spatial reversal performance in rats with selective lesions to these structures were the same as those used in the present investigation.

\section{DISCUSSION}

\section{Spatial Reversal Learning}

Sensory basis. Although the present study was not designed to investigate the sensory basis of spatial learning in the rat, the abnormal performance of the group sustaining both olfactory bulbectomy and enucleation of the eyes makes it necessary to comment on this important issue. As noted earlier, olfactory bulbectomy combined with peripheral blinding led to substantially poorer reversal-learning scores (even on the first reversal) than either surgical intervention alone. These results, of course, are reminiscent of the classical findings of Honzik (1936), who reported a similar pattern of results in connection with acquisition of a 14-cul elevated maze. Honzik interpreted his results in terms of the disproportionate dependence on olfactory cues by blinded rats coupled with a slight, but enduring, 


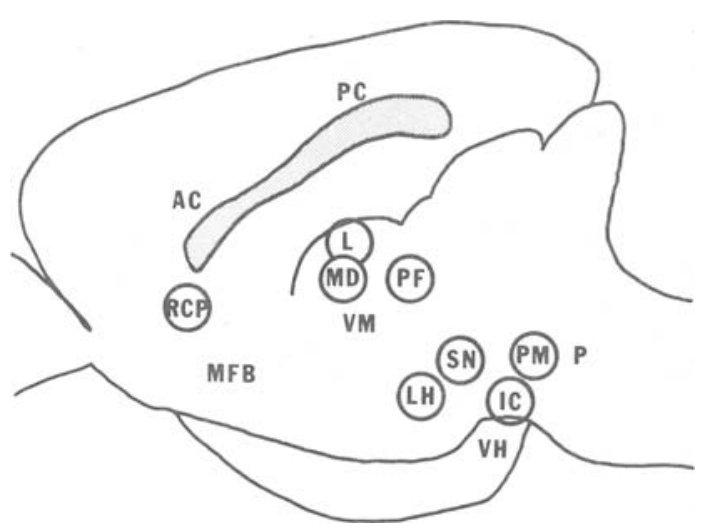

Figure 9. Schematic drawing of a parasagittal section of the rat brain showing the relative positions of those lesion placements producing marginally significant (unencircled capital letters) and significant (encircled capital letters) deficits in retention of individual reversal problems. (See Figure 8 for abbreviations.)

depression of neocortical functioning subsequent to privation of the two sensory systems.

In light of the foregoing observations, it might be suggested that the two most relevant sensory cues involved in T-maze reversal learning lie within the visual and olfactory domains. This would seemingly explain the slight reversal-learning loss associated with either enucleation of the eyes or olfactory bulbectomy and the gross reversal-learning loss associated with combined enucleation and bulbectomy. This explanation is not very appealing for a number of reasons. First of all, no salient intramaze or extramaze visual or olfactory cues differentiating the left alley from the right alley were available to the rats studied in the current experiment. Second, in the process of learning a simple visual discrimination or a simple odor discrimination under conditions of escape-avoidance of footshock and punishment for errors, control (and the majority of brain-damaged) rats usually exhibit vicarious trial-and-error responses in the former situation (Thompson, 1969) and periods of sniffing responses in the latter situation (Thompson, 1980). These stimulus-sampling behaviors were rarely observed in the T-maze reversal situation of the present experiment. In fact, most of the control and experimental rats appeared to have made their choices of the left or right arm prior to reaching the choice point. And finally, there is evidence that left-right differentiation in rodents has a vestibular (Douglas, Clark, Erway, Hubbard, \& Wright, 1979), as well as a proprioceptive-kinesthetic (Thompson, Hale, \& Bernard, 1980), basis and that these animals may utilize these internal cues to solve spatial tasks in the absence of visual cues (Zoladek \& Roberts, 1978).

One alternative explanation of what might be termed the "Honzik effect" is based on the theory of O'Keefe and Nadel (1978) as it relates to spatial discrimination reversals and the hippocampus. These investigators have proposed that hippocampallesioned rats show a spatial reversal-learning deficit because they are predisposed to use "orientation" hypotheses rather than the "place" hypotheses that normal rats would typically use in a maze situation. Could it be that rats subjected to both enucleation of the eyes and olfactory bulbectomy are also prone to use orientation hypotheses and, as a result, exhibit dramatic impairments in spatial reversal (and complex maze) learning?

While this explanation of the Honzik effect is incomplete to the extent that it fails to grapple with the neurobiological mechanism responsible for the shift from place to orientation strategies, its value lies in the ease with which it can be tested. For example, those active and passive avoidance tasks found to be sensitive to the presence of hippocampal damage (Black, Nadel, \& O'Keefe, 1977) should also be sensitive to the presence of combined bulbectomy and enucleation.

General findings. Earlier studies have reported spatial reversal-learning impairments in rats prepared with lesions to the cerebral cortex (Divac, 1971; Thorne \& Thompson, 1970), corpus striatum (Divac, 1971; Hannon \& Bader, 1974; Kolb, 1977), limbic system (Kimble \& Kimble, 1965; Samuels, 1972; Thompson \& Langer, 1963), thalamus (Kolb, 1977; Means, Hershey, Waterhouse, \& Lane, 1975; Thorne \& Thompson, 1970), hypothalamus (Thompson \& Langer, 1963), or midbrain (Thorne \& Thompson, 1970). The results of the present experiment not only confirm these findings, but attest to the unusually high degree of sensitivity of the spatial reversal-learning task to the presence of brain damage in the albino rat. In a related series of studies, for example, visual reversal learning was not subject to interference by lesions of the olfactory bulbs, parietal cortex, anterior cingulate cortex, rostral caudoputamen, dorsal hippocampus, ventral hippocampus, septofornix, nucleus accumbens, rostral medial forebrain bundle, medial preoptic-supraoptic area, or ventromedial hypothalamus (Thompson, 1982a, 1982b, Note 1). In contrast, learning of the spatial reversal series (or even the first reversal problem) studied in the present experiment was significantly impaired by discrete lesions placed in any one of the foregoing structures and in most other structures of the brain as well. It should be pointed out that the foregoing results are not entirely unexpected in view of earlier reports demonstrating that spatial (maze) habits are considerably less localized at cortical and subcortical levels than visual habits (Lashley, 1950; Thompson, 1982c).

Another interesting finding deals with the equiv- 
alent reversal-learning deficits associated with selective lesions to the occipital, parietal, and frontal (motor) areas of the cerebral cortex. This outcome is reminiscent of the classical findings reported by Lashley (1929) in connection with acquisition of a complex maze, but differs to the extent that no significant relationship was found between the severity of the reversal-learning loss and the magnitude of neocortical damage. Apparently, the singleunit T-maze reversal habit is not of sufficient difficulty to be sensitive to varying amounts of neocortical damage, but is of sufficient difficulty to be sensitive to the presence of neocortical damage. Parenthetically, the finding that the occipital group showed a significantly greater reversal-learning deficit than the blinded group constitutes another line of evidence supporting Lashley's notion that the occipital cortex has a nonvisual function (see Thompson, 1982d).

Despite the sensitivity of the T-maze reversal problem to the presence of brain damage and the equivalent learning losses associated with lesions to different areas of the neocortex, it cannot be concluded that each part of the rat brain contributes equally to the formation of spatial reversal tasks. As shown in Table 1, certain lesion placements produced substantially greater reversal-learning deficits than other lesion placements. At the same time, however, there appears to be a limit to the degree that the spatial reversal-learning process can be localized within the rat brain. The finding that critical foci for spatial reversal-learning deficits inhabit portions of the tel-, di-, mes-, and metencephalon (see Figure 8) favors the view that a widespread neuroanatomical mechanism underlies this class of learning.

Comparison with visual reversal learning. By comparing the data of the present experiment on spatial reversal learning with those recently obtained on visual (white-black) reversal learning (Thompson, 1982a, 1982b, Note 1), some insights into the brain mechanisms subserving spatial and visual reversal learning in particular and reversal learning in general can be achieved. Any conclusions drawn from this comparison, however, must be viewed with caution, since a number of methodological differences exist between the two studies. While the experimental procedures overlapped at many points (subjects of the same strain and of comparable age and previous experience, lesions of comparable size and location, motive of escape-avoidance of footshock, punishment for errors, comparable intertrial intervals, and the same criterion of learning), they differed with respect to: (1) the difficulty of the problem (the white-black discriminations were substantially more difficult to master than the spatial discriminations); (2) the degree of distributed practice (learning of each visual problem required multiple training sessions, while learning of each spatial problem required only one training session); (3) the apparatus (a Thompson-Bryant discrimination box was used for the visual problems, while a single-unit $\mathrm{T}$-maze was used for the spatial problems); (4) the number of reversals (only one visual reversal problem was studied as compared with eight spatial reversal problems); and (5) the definition of a critical area for reversal learning (in the visual study, the critical areas were derived from those brain-damaged groups that were significantly impaired in learning the reversal problem or showed a significant-or marginally significant-reversal deficit $^{2}$; in the spatial study, the critical areas were derived from those brain-damaged groups that committed at least 2.5 errors/reversal).

Figure 10 depicts the results of this comparison. One group of structures appears to be associated mainly with a visual reversal deficit (areas marked by vertical lines), a second group with a spatial reversal deficit (areas marked by horizontal lines), and a third group with both visual and spatial reversal deficits (shaded areas). (The median raphe and pontomesencephalic reticular formation areas are not included in Figure 10 because of incomplete information on the effects of lesions to these structures on visual reversal learning.)

It seems clear from inspection of Figure 10 that some structures related to the basal ganglia (globus pallidus, ventrolateral thalamus, substantia nigra, and red nucleus) are primarily concerned with visual reversal learning, whereas other structures related to the limbic forebrain (anterior cingulate cortex, dorsal hippocampus, septofornix, lateral preoptic-supraoptic area, medial preoptic-supraoptic area, and ventromedial hypothalamus) are primarily

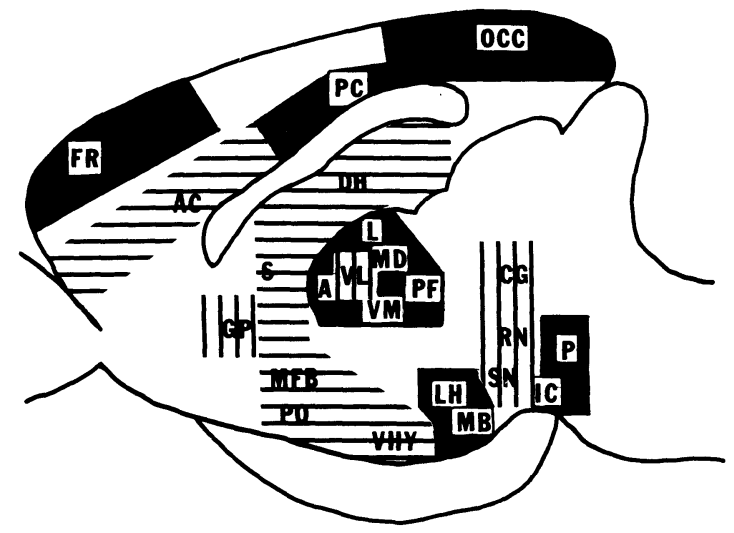

Figure 10. Schematic drawing of a parasagittal section of the rat brain showing the relative positions of those brain sites mainly concerned with visual reversal learning (areas marked by heavy vertical lines), spatial reversal learning (areas marked by heavy horizontal lines), and both visual and spatial reversal learning (shaded areas). (See Figure 8 for abbreviations.) 
concerned with spatial reversal learning. On the other hand, the distribution of those structures critical for both visual and spatial reversal learning does not appear to be organized along the lines of any well-defined neuroanatomical (or neuropharmacological) pathway, although a major portion does overlap with certain specific and nonspecific thalamocortical and/or corticothalamic systems.

The extent to which those brain sites concerned with both visual and spatial reversal learning are also concerned with other sensory reversal-learning tasks remains to be seen. There is some evidence, for example, that the occipital cortex may participate only in spatial and visual reversal learning, while the lateral frontal cortex may play a more nonspecific role in reversal learning. This suggestion is based on the report of Gabriel, Freer, and Finger (1979), which showed that frontal lesions, but not occipital lesions, impaired tactile reversal learning in rats.

The question also arises concerning the extent to which the results shown in Figure 10 can be generalized to other mammalian forms. One line of evidence favoring at least some degree of generality comes from the findings that monkeys with hippocampal (Jones \& Mishkin, 1972; Mahut, 1971) or fornix column (Mahut, 1972) damage show spatial reversal-learning impairments, but not visual (object) reversal-learning impairments. Even more striking are the observations recently reported by Oscar-Berman and Zola-Morgan (1980). They found that alcoholic Korsakoff patients (presumed to have mammillary body and/or medial thalamic lesions) exhibited both a spatial and a visual reversallearning loss, while patients with Huntington's disease (presumed to have basal ganglia lesions) exhibited a visual reversal-learning loss only.

\section{Spatial Reversal Retention}

The present data on retention (retentiveness) of individual spatial reversal problems are consistent with those of Huppert and Piercy (1979) to the extent that abnormal forgetting is a function of the site of brain damage and brain-damaged subjects exhibiting slow learning do not necessarily exhibit rapid forgetting. Thus, while certain brain-damaged rats (those with lesions of the mediodorsal thalamic nucleus, posterolateral hypothalamus, or pontomesencephalic reticular formation) were found to be markedly impaired in both learning and retention, other brain-damaged rats (those with lesions of the dorsal hippocampus, septofornix, anterior thalamus, mammilary bodies, or median raphe) were found to be markedly impaired in learning only.

This apparent dissociation between learning and retention disorders is further demonstrated by the finding that none of the correlations between the number of learning errors and the number of retention errors approached statistical significance in any one of the 27 brain-damaged groups involved in this study, despite the fact that a marginally significant positive relationship was evident in the control group.

If a spatial reversal learning defect is indeed completely independent of a spatial reversal retention defect, then it should be possible to observe the latter in the absence of the former. While this pattern of results was not evident in any of the experimental groups investigated in the current study, it has been observed in aged rats (Zornetzer, Thompson, \& Rogers, 1982). [Interestingly, a similar pattern of results has been reported in hippocampal-lesioned rodents tested on a visual discrimination (Sara \& David-Remacle, 1981) and a passive avoidance (Zornetzer et al., 1977) habit.]

In light of these findings, it is reasonable to propose that there may be at least three classes of learning (amnesic) disorders observed on any given task: (1) a learning impairment only, (2) a retention impairment only, and (3) both learning and retention impairments. Such a classification implies that the neurobiological events underlying learning are quite different from those underlying retention. This, of course, is reminiscent of the "dual-trace" hypothesis, which is a cornerstone within the framework of a consolidation theory of memory (see McGaugh \& Herz, 1972). Evidence that some kind of consolidation process may be taking place within the spatial reversal-learning situation has been reported earlier (Thompson, 1958; Thompson, Langer, \& Rich, 1964; Thompson \& Yang, 1982).

As shown in Figure 9, no particular area of the neocortex seems to play a significant role in retention of individual spatial reversal problems in the rat. More surprisingly, the dorsal hippocampus also seems to contribute little to the retention of spatial reversal problems. This latter finding fails to confirm earlier observations (Thompson, 1981) that dorsal hippocampal-lesioned rats show rapid forgetting of spatial reversal problems. This discrepancy is more than likely due to the fact that the earlier study utilized a weak criterion of learning (five consecutive correct responses), while the current experiment employed a more stringent criterion of learning involving a run of correct responses having a probability of occurrence of at least .05 . [It has previously been noted that a weak criterion of learning is associated with poor retention of spatial reversal problems even in normal rats (Thompson, 1959).] However, the finding that mammillary-body-lesioned rats in the earlier study failed to exhibit rapid forgetting under the condition of a weak criterion of learning suggests that the dorsal hippocampus may play an important 
role in maintaining "underlearned" spatial information in store.

The map of critical foci for spatial reversalretention deficits shown in Figure 9 does not appear to overlap with any recognizable neuroanatomical or neuropharmacological system. The limbic forebrain, which is clearly implicated in spatial reversal learning (see Figure 8), seems to contribute little to long-term retention of spatial reversal problems. In a previous report (Thompson \& Yang, 1982), it was suggested that the nigral-parafascicular-striatal complex may participate in some way in the maintenance of spatial information in store. It now appears, however, that this complex is but a part of a larger neurological system that may serve this function. Conceivably, further insight into the organization of those brain sites concerned with retention of spatial reversal problems in particular. and retention in general will emerge when data become available on those brain sites concerned with retention of visual reversal problems.

\section{REFERENCE NOTE}

1. Thompson, R. Unpublished observations, 1982.

\section{REFERENCES}

Black, A. H., NAdel, L., \& O'Keefe, J. Hippocampal function in avoidance learning and punishment. Psychological Bulletin, 1977, 84, 1107-1129.

Divac, I. Frontal lobe system and spatial reversal in the rat. Neuropsychologia, 1971, 9, 175-183.

Douglas, R. J., Clark, G. M., Erway, L. C., Hubbard, D. G., \& Wright, C. G. Effects of genetic vestibular defects on behavior related to spatial orientation and emotionality. Journal of Comparative and Physiological Psychology, 1979, 93, 467-480.

Gabriel, S., Freer, B., \& Finger, S. Brain damage and the overlearning reversal effect. Physiological Psychology, 1979, 7, 327-332.

Grant, D. A. New statistical criteria for learning and problem solution in experiments involving repeated trials. Psychological Bulletin, 1946, 43, 558-561.

Hannon, R., \& Bader, A. A comparison of frontal pole, anterior median and caudate nucleus lesions in the rat. Physiology \& Behavior, 1974, 13, 513-521.

HonzIK, C. H. The sensory basis of maze learning in rats. Comparative Psychology Monographs, 1936, 13, 1-113.

Huppert, F. A., \& Piercy, M. Normal and abnormal forgetting in organic amnesia: Effect of locus of lesion. Cortex, 1979, $15,385-390$.

Jones, B., \& Mishkin, M. Limbic lesions and the problem of stimulus-reinforcement associations. Experimental Neurology, 1972, 36, 362-377.

Kimble, D. P., \& Kimble, R. J. Hippocampectomy and response perseveration in the rat. Journal of Comparative and Physiological Psychology, 1965, 60, 474-476.

KolB, B. Studies on the caudate-putamen and the dorsomedial thalamic nucleus of the rat: Implications for mammalian frontal-lobe functions. Physiology \& Behavior, 1977, 18, 237-244.

LASHLEY, K. S. Brain mechanisms and intelligence. Chicago: University of Chicago Press, 1929.

LASHLEY, K. S. In search of the engram. In Symposium of the
Society for Experimental Biology (No. 4). Cambridge: Cambridge University Press, 1950.

MaнUt, H. Spatial and object reversal learning in monkeys with partial temporal lobe ablations. Neuropsychologia, 1971, 9, 409-424.

Mahut, H. A selective spatial deficit in monkeys after transection of the fornix. Neuropsychologia, 1972, 10, 65-74.

McGaugh, J. L., \& Herz, M. J. Memory consolidation. San Francisco: Albion, 1972.

Means, L. W., Hershey, A. E., Waterhouse, G. J., \& Lane, C. J. Effects of dorsomedial thalamic lesions on spatial discrimination reversal in the rat. Physiology \& Behavior, 1975, 14, 725-729.

O'KeEfe, J., \& NADEL, L. The hippocampus as a cognitive map. Oxford: Clarendon Press, 1978.

Oscar-Berman, M., \& Zola-Morgan, S. M. Comparative neuropsychology and Korsakoff's syndrome. I. Spatial and visual reversal learning. Neuropsychologia, 1980, 18, 499-512.

Runnels, L. K., Thompson, R., \& Runnels, P. Near-perfect runs as a learning criterion. Journal of Mathematical Psychology, 1968, 5, 362-368.

Samuels, I. Hippocampal lesions in the rat: Effects on spatial and visual habits. Physiology \& Behavior, 1972, 8, 10931098.

SARA, S. J., \& David-Remacle, M. Discrimination avoidance learning in hippocampal and cortical rats: Acquisition rate, behavioral strategies, and long-term retention. Physiological Psychology, 1981, 9, 37-48.

Siggel, S. Nonparametric statistics. New York: McGraw-Hill, 1956.

Seuire, L. R. Two forms of amnesia identified through an analysis of forgetting. Society for Neuroscience Abstracts, 1980, $6,850$.

Thompson, R. The effect of intracranial stimulation on memory in cats. Journal of Comparative and Physiological Psychology, 1958, 51, 421-426.

Thompson, R. Transient memory in albino rats. Science, 1959, 129, 842-843.

Thомpson, R. Localization of the "visual memory system" in the white rat. Journal of Comparative and Physiological Psychology Monograph, 1969, 69, Part 2, 1-29.

Thompson, R. A behavioral atlas of the rat brain. New York: Oxford University Press, 1978.

Thompson, R. Odor discrimination performance as affected by cortical, thalamic and cerebellar lesions in rats. Physiology \& Behavior, 1980, 24, 797-800.

Thompson, R. Rapid forgetting of a spatial habit in rats with hippocampal lesions. Science, 1981, 212, 959-960.

Thompson, R. Brain lesions impairing visual and spatial reversal learning in rats: Components of the "general learning system" of the rodent brain. Physiological Psychology, 1982, 10, 186198. (a)

Thомpson, R. Impaired visual and spatial reversal learning in brain-damaged rats: Additional components of the "general learning system" of the rodent brain. Physiological Psychology, 1982, 10, 293-305. (b)

Thompson, R. Functional organization of the rat brain. In J. Orbach (Ed.), Neuropsychology after Lashley. Hillsdale, N.J: Erlbaum, 1982. (c)

Thompson, R. Evidence that the occipital cortex also functions in place learning in rats. In A. Ajmone Marsan \& H. Matthies (Eds.), Neuronal plasticity and memory formation. New York: Raven Press, 1982. (d)

Thompson, R., \& Langer, S. K. Deficits in position reversal learning following lesions of the limbic system. Journal of Comparative and Physiological Psychology, 1963, 56, 987-995.

Thompson, R., \& Y ANG, S. Retention of individual spatial reversal problems in rats with nigral, caudoputamenal, and reticular formation lesions. Behavioral and Neural Biology, $1982,34,98-103$. 
Thompson, R., Hale, D. B., \& Bernard, B. B. Brain mechanisms concerned with left-right differentiation in the white rat. Physiological Psychology, 1980, 8, 309-319.

Thompson, R., Kao, L., \& Yang, S. Rapid forgetting of individual spatial reversal problems in rats with parafascicular lesions. Behavioral and Neural Biology, 1981, 33, 1-16.

Thompson, R., Langer, S. K., \& Rich, I. Lesions of the limbic system and short-term memory in albino rats. Brain, 1964, 87, 537-542.

Thorne, B. M., \& Thompson, R. Position reversal learning as affected by occipital, posterior thalamic, and rubral lesions in the white rat. Psychonomic Science, 1970, 18, 189-190.

ZoladeK, L., \& Roberts, W. A. The sensory basis of spatial memory in the rat. Animal Learning \& Memory Behavior, 1978, 6, 77-81.

Zornetzer, S. F., Gold, M. S., \& Boast, C. A. Neuroanatomic localization and the neurobiology of sleep and memory. In R. R. Drucker-Colin \& J. L. McGaugh (Eds.), Neurobiology of sleep and memory. New York: Academic Press, 1977.
Zornetzer, S. F., Thompson, R., \& Roberts, J. Rapid forgetting in aged rats. Behavioral and Neural Biology, 1982, $36,49-60$.

\section{NOTES}

1. These data were referred to in the two previous studies (Thompson, 1982a, 1982b) concerned with the identification of the components of the "general learning system" of the rodent brain.

2. A reversal deficit for a given brain-damaged group was considered to be present if that group committed proportionately more errors (or required proportionately more trials) in learning the reversal problem, as opposed to the original problem, than the controls.

(Manuscript received December 13, 1982; revision accepted for publication January $31,1983$. 